\title{
EBSP-based Crystal Plasticity FEM Simulation of Microscopic Stress Distribution in Weld Metal*
}

\author{
by Yoshiki Mikami**, Keisuke Sogabe*** and Masahito Mochizuki**
}

\begin{abstract}
In order to evaluate the occurrence of cracks such as SCC, it is important to estimate microscopic stress distribution. The microscopic stress distribution is generated due to microscopic inhomogeneity. In this study a numerical simulation method to evaluate the effect of crystal orientation was proposed. The method consists of measurement of crystal orientation by EBSP method and microscopic stress analysis based on the theory of crystal plasticity considering EBSP measurement result. The method was applied to the microscopic stress calculation of the weld metal band electrode submerged arc welding of Ni-base alloy. It was demonstrated that microscopic stress concentration occurs around the grain boundary of the weld metal.
\end{abstract}

Key Words: EBSP, crystal orientation, crystal plasticity, microscopic stress, numerical simulation

\section{Introduction}

It is important to prevent the occurrence of cracks such as SCC for safe operation of nuclear power plants. Evaluation of stress distribution in microscopic scale is a key technology to predict occurrence of cracks. The microscopic stress distribution arises mainly from crystal orientation and grain shape of polycrystalline structure. In this study, EBSP-based crystal plasticity finite element method, a method to calculate microscopic stress distribution in polycrystalline materials taking into consideration crystal orientation and grain shape was proposed. The information of grain aggregate was obtained by EBSP (electron backscattering pattern) measurement technique. The measurement results were introduced into a finite element model and were used in the following microscopic stress simulation. In the calculation of microscopic stress, theory of crystal plasticity was incorporated to the finite element analysis method in order to take into consideration of the effect of crystal orientation. Finally, the proposed method was applied to the calculation of microscopic stress distribution in weld metal.

\section{EBSP-based Crystal Plasticity FEM}

\subsection{EBSP Analysis of Weld Metal}

The EBSP-based crystal plasticity FEM includes the

${ }^{*}$ Received: 2010.11 .11

** Member, Division of Materials and Manufacturing Science, Graduate School of Engineering, Osaka University

*** Student Member, Division of Materials and Manufacturing Science, Graduate School of Engineering, Osaka University measurement of crystal orientation and microscopic stress simulation considering theory of crystal plasticity. At first, weld pieces of Ni-base alloy were fabricated by band electrode submerged arc welding as reported by Nishikawa et al ${ }^{1)}$. Specimens were extracted from the weld metal of fabricated weld piece and measurement of crystalline orientation on the surface of the weld bead was performed by EBSP-OIM method. The IPF (inverse pole figure) map of the surface of Ni-base alloy weld metal is shown in Fig. 1. Generally, the columnar grain grows along maximum temperature gradient direction; the crystal orientation in weld metal becomes anisotropic. Especially for the band electrode submerged arc welding, heat input is relatively high, therefore the tendency becomes more remarkable. As a result, the columnar grain grows towards the bead surface direction, so that $\left(\begin{array}{lll}1 & 0 & 0\end{array}\right)$ direction is mainly observed on the bead surface. Consideration of such microscopic inhomogeneity is important in the evaluation of microscopic stress distribution in weld metal.

\subsection{Microscopic Stress Analysis Method Incorporating Crystal Plasticity}

The inelastic deformation of crystalline materials is caused by slip deformation of crystals. In polycrystalline materials, each crystal grain has its own crystal orientation, which causes inhomogeneous deformation and finally leads to the formation of a microscopic distribution of stresses and strains. Constitutive modeling theories for crystal plasticity were first proposed by Taylor ${ }^{2)}$, and followed by various researchers. In this study, the formulation presented by Pierce, Asaro and Needleman ${ }^{3)}$ was adopted, and was incorporated into the commercial multi-purpose finite element analysis software Abaqus (version 6.9-1) through user subroutine UMAT ${ }^{4,5}$. 
In this study, face-centered cubic (FCC) materials such as austenitic stainless steels and nickel-base alloys were modeled according to crystal plasticity theory. The modeling could also be extended to body-centered cubic materials such as ferritic steels. For FCC material, twelve slip systems were introduced into the formulation. Slip deformation occurs due to shear stresses acting on a slip plane in a slip direction. A rate-dependent crystalline slip model was adopted for the slip deformation. The shear strain rate on slip system $\dot{\gamma}^{(\alpha)}$ depends on the resolved shear stress $\tau_{i j}{ }^{\alpha}$. The shear stress is determined by Schmid's law,

$$
\tau_{i j}^{(\alpha)}=P_{i j}^{(\alpha)} S_{i j}
$$

where $P_{i j}^{(\alpha)}$ : Schmid tensor, $S_{i j}:$ stress tensor.

The shear slipping rate of the slip system a is calculated by the following power law.

$$
\dot{\gamma}^{(\alpha)}=\dot{a}^{(\alpha)}\left(\frac{\tau^{(\alpha)}}{g^{(\alpha)}}\right)\left|\frac{\tau^{(\alpha)}}{g^{(\alpha)}}\right|^{n-1}
$$

where, $\dot{\gamma}^{(\alpha)}$ : the shear strain rate on slip system $\alpha, \dot{a}^{(\alpha)}$ : the reference strain rate on slip system $\alpha, \tau_{i j}{ }^{(\alpha)}$ : the resolved shear stress on slip system $\alpha, g^{(\alpha)}$ : a function describing the current strength of slip system a, $n$ : the rate sensitivity exponent.

Equation (2) describes the relationship between $\tau$ and $g$ in the case that the shear strain rate on slip system $\dot{\gamma}^{(\alpha)}$ equals $\dot{a}^{(\alpha)}$. When $n$ approaches $\infty$, Eq. (2) becomes more rate-independent. Function $g^{(\alpha)}$, which describes the resistance of a crystal against slip deformation, is dependent on the total slip deformation, as in the following equation.

$$
g^{(\alpha)}=g^{(\alpha)}(\gamma), \gamma=\sum_{\alpha} \int_{0}^{t}\left|\dot{\gamma}^{(\alpha)}\right| d t
$$

Strain hardening is characterized by the evolution of function $g^{(\alpha)}$, i.e., $\dot{g}^{(\alpha)}$,

$$
\dot{g}^{(\alpha)}=\sum_{\beta} h_{\alpha \beta} \dot{\gamma}^{(\beta)}
$$

where $h_{\alpha \beta}$ : the slip hardening moduli.

In Eq. (4), $\dot{g}^{(\alpha)}$, which is the evolution of function $g^{(\alpha)}$, is related to the hardening of all slip systems; therefore, it is expressed as the summation of all slip systems. The hardening moduli $h_{a \alpha}$ and $h_{a \beta}(\alpha \neq \beta)$ are called self and latent hardening. The self-hardening moduli used is,

$$
h_{\alpha \alpha}=h(\gamma)=h_{0} \operatorname{sech}^{2}\left|\frac{h_{0} \gamma}{\tau_{s}-\tau_{0}}\right|
$$

where $h_{0}$ : the initial hardening modulus, $\tau_{0}$ : the yield stress which equals the initial value of the current strength, $\tau_{\mathrm{s}}$ : the saturation strength.

The value of $\tau_{0}$ is $g^{(\alpha)}(0)$ for each slip system $\alpha$, and $g$ is already presented in Eq. (3). The latent hardening moduli are given by

$$
h_{\alpha \beta}=q h(\gamma) \text { for } \alpha \neq \beta
$$

The parameter $q$ represents the level of latent hardening in comparison with self hardening. In this study, $q$ was set to 1 : the Taylor-type isotropic hardening.

Under the above formulation, plastic shearing occurs when the resolved shear stress on a slip system becomes a non-zero value.

\section{Microscopic Stress Simulation of Weld Metal by EBSP-based Crystal Plasticity FEM}

\subsection{Microscopic Stress Analysis Model}

The obtained EBSP measurement result is shown in Fig. 1. Based on the EBSP result, a simulation model for the calculation of microscopic stress distribution was generated. The grain shape was identical to the EBSP measurement result. The shape is extruded through thickness direction, as columnar grain grows along surface direction in the weld metal of the fabricated weld piece. The simulation model corresponding to EBSP measurement result is shown in Fig. 2. The measured crystal orientation distribution was introduced into a finite element model. The crystal orientation could be uniquely determined by using Euler angle expressed by $\phi_{1}, \Phi$ and $\phi_{2}$, data available from EBSP analysis software.

For the generated finite element model, tensile displacement was imposed as boundary condition in order to simulate the microscopic stress distribution. The applied tensile displacement corresponds to $1 \%$ global tensile strain in longitudinal direction of the simulation model. 

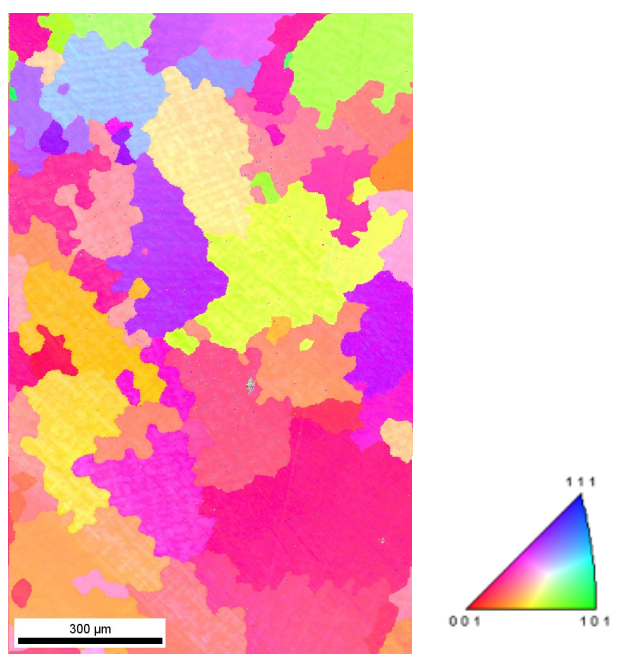

Fig. 1 IPF map of Ni-base alloy weld metal.

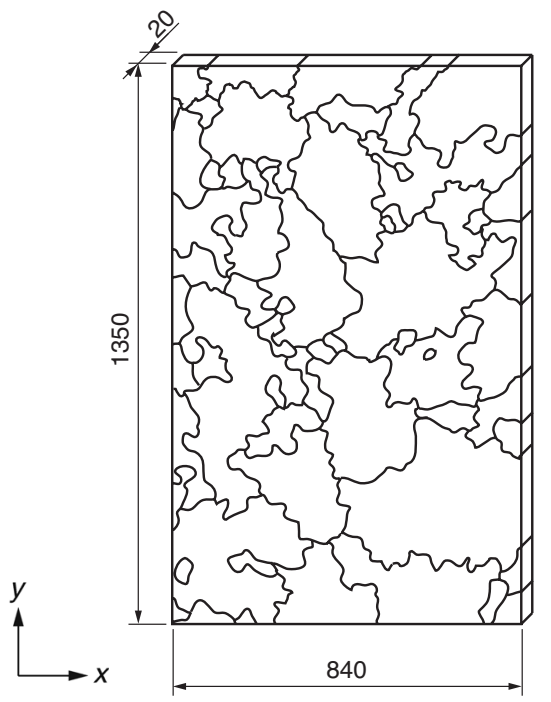

Fig. 2 Simulation model of microscopic stress in weld metal.

\subsection{Material Properties Used}

\section{Related to Crystal Plasticity in the Simulation}

In order to perform microscopic stress simulation, it is needed to determine the material properties that characterize crystal plasticity should be determined. However, it is quite difficult to measure properties used in the theory of crystal plasticity, $h_{0}, \tau_{0}$, $\tau_{\mathrm{s}}$ for example, presented in the section 2.2. Therefore in this study, the material properties used in crystal plasticity were determined as follows.

Elastic anisotropy was not taken into consideration in this study. It was also confirmed that the effect of the elastic anisotropy was little in the simulation model used. The elastic material properties were isotropic in this model, i.e., elastic modulus $E=190 \mathrm{GPa}$, Poison's ratio $v=0.3$.

Slip deformation properties in crystal plasticity theory should

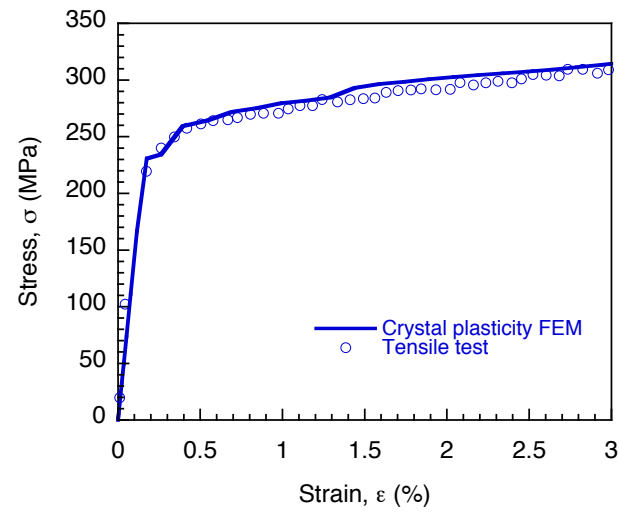

Fig. 3 Comparison of stress-strain curves between crystal plasticity FEM and tensile test.

be defined to perform the numerical simulation of microscopic stress distribution. This is equivalent to define a hardening law of slip system. In order to determine parameters involved in the theory of crystal plasticity, a simulation of tensile test of an aggregate of 100 grains with random crystal orientation distribution was performed. By applying tensile displacement to the model, macroscopic stress-strain curve could be obtained. The macroscopic stress-strain curve is affected by parameters of crystal plasticity. Therefore, the parameters were determined to reproduce the experimentally obtained stress-strain curve by the simulation result of an aggregate of 100 grains model. The parameters $h_{0}, \tau_{0}, \tau_{\mathrm{s}}$, determined by 100 grains model were, 100 , 75 , 95, respectively. Fig. 3 is the comparison of macroscopic stress-strain curves between the experiment and the simulation.

\subsection{Simulation Result of Microscopic Stress Distribution}

Microscopic stress distribution in columnar grain scale in weld metal was calculated by the proposed analysis method incorporating crystal plasticity theory. Distribution of microscopic stress obtained by the simulation is shown in Fig. 4. The stress along loading direction (vertical direction) is plotted in the figure. In Fig. 4, the applied strain level is $1 \%$, therefore the average stress observed in the model is about $250 \mathrm{MPa}$, which is corresponding to the macroscopic stress-strain curve in Fig. 3. Microscopically, as seen from the figure, the distribution of stress is inhomogeneous as the crystal plasticity was incorporated to the simulation model and grain orientation was taken into consideration. 


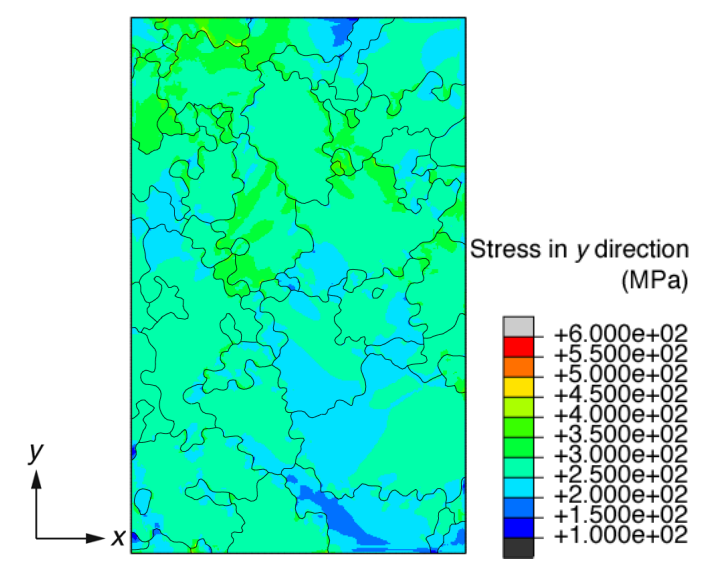

Fig. 4 Simulation result of microscopic stress distribution in weld metal of Ni-base alloy.

\section{Summary}

In this study, EBSP-based crystal plasticity FEM simulation: an analysis method applicable to the calculation of microscopic stress distribution in weld metal based on EBSP measurement results was presented. The method was applied to the calculation of microscopic stress distribution in Ni-base alloy band electrode submerged arc weld metal. Microscopic stress distribution was obtained by the proposed simulation method and it was demonstrated that microscopic stress concentration occurs around grain boundaries.

\section{Acknowledgements}

The authors appreciate the support for the fabrication of weld specimen and the discussion in the modeling of weld metal by Dr. Satoru Nishikawa (JAPEIC, Japan Power Engineering and Inspection Corporation).

This study was supported by Priority Assistance for the Formation of Worldwide Renowned Centers of Research - The Global COE Program (Project: Center of Excellence for Advanced Structural and Functional Materials Design) from the Ministry of Education, Culture, Sports, Science and Technology (MEXT), Japan.

\section{Reference}

1) S. Nishikawa, Y. Horii and K. Ikeuchi: Effects of Carbon and Niobium Contents on Stress Corrosion Cracking Susceptibility of Shielded Metal Arc Weld Metals for 600 Type Alloy in High Temperature Pressurized Pure Water, Quarterly Journal of the Japan Welding Society, .27-3 (2009), pp. 247-260. (in Japanese)

2) G. I. Taylor: Plastic Strain In Metals, Journal of the Institute of Metals, 62 (1938), 307-324.

3) D. Pierce, R. J. Asaro and Needleman: Material rate dependence and localized deformation in crystalline solids, Acta Metallurgica, 31-12 (1983), 1951-1976.

4) Abaqus User Subroutines Reference Manual Version 6.9, (2009).

5) Y. Huang: A User-material Subroutine Incorporating Single Crystal Plasticity in the Abaqus Finite Element Program, Harvard University Mech Report 178, (1991). 